\title{
Editorial: Mobile Networks in the Era of Big Data
}

\section{Vijayakumar Varadarajan ${ }^{1} \cdot$ Venkataraman Neelanarayanan $^{1} \cdot$ Ron Doyle $^{2} \cdot$ Imad Fakhri Al-Shaikhli $^{3} \cdot$ Sven Groppe $^{4}$}

Published online: 29 June 2019

(C) Springer Science+Business Media, LLC, part of Springer Nature 2019

\section{Editorial:}

There is an exceptional rupture of research activities in big data, which illustrate enormous potential in making decisions, optimizing operations, mitigating security threats and also in a variety of fields, such as retail, advertisement, manufacturing, healthcare and insurance. Data from Mobile devices and networks like smart phones, cellular networks, Sensor networks, Vehicular Networks is an important source of big data. Thus, Mobile networks play a critical role in big data generation, delivery, and processing. Big Data will have profound impacts on the design and operation of Mobile networks.

We received 46 paper submissions, from which 7 submissions have been transferred and 8 papers withdrawn. After a rigorous single blinded peer review process only 14 papers have been accepted and 17 papers have been rejected, i.e., our acceptance rate is about $45 \%$ not considering the transferred and withdrawn submissions. Hence, this special issue features 14 high quality research papers that can demonstrate proofs-of-concept, services, solutions for research challenges, case studies, analytics, real world examples and successful deliveries of Big Data and Mobile Networks.

\section{Vijayakumar Varadarajan}

vijayakumar.v@vit.ac.in

Venkataraman Neelanarayanan

neelanarayanan.v@vit.ac.in

Ron Doyle

rdoyle@alumni.duke.edu

Imad Fakhri Al-Shaikhli

imadf@iium.edu.my

Sven Groppe

groppe@ifis.uni-luebeck.de

Vellore Institute of Technology, Chennai, India

2 Continuous Delivery Architecture, CA Technologies, New York, NY, USA

3 International Islamic University, Malaysia, Gombak, Malaysia

4 University of Lübeck, Lübeck, Germany
The first article [1] deals with mobile communication, where base stations handle mobile devices. The process of assigning mobile nodes to other base stations (because they handle already too many mobile devices or the devices leave the supported area) is called heterogeneous handover, which is needed for seamless integration of networks. The contribution [1] proposes a vertical handover for LTE based on various signal measures like the enhanced weighted sum method for handover decision making. Finally, relevant criteria are utilized and their benefits analyzed.

The current state-of-the-art textual password authentication mechanisms are prone to eavesdropping, dictionary attacks and shoulder surfing. Hence, the second article [2] proposes a secure group communication scheme between group members using graphical passwords, which are quite secure using the elliptic curve cryptography technique for key distribution and are easier to remember in comparison to textual passwords. Furthermore, the proposed scheme ensures negligible overhead in terms of communication and computation.

Patients and healthcare seekers exchange their knowledge on health-related issues on online healthcare community discussion forums. The contribution [3] analyzes these discussion forums as e.g. Medhelp for pregnancy data by utilizing metrics like quality of information, emotional support, source trustworthiness, replier competition and recipient involvement, and proposes a knowledge contribution model.

Synthetic Aperture Radar (SAR) images are often degraded due to noise (being an undesirable fluctuation in a random portion of the image), blur (reducing the object visibility) and artifacts (like the shadowing effect). The contribution in [4] provides an analysis for the impact of image artifacts (like shadow effects) in real time images for the purpose of detecting and removing the shadowing effect from very high resolution (VHR) SAR images and aerial view Images.

Targeted anti-forensic attacks eliminate statistical footprints left by contrast enhancement on histograms, such that image modifications become undetectable, and also introduce anomalies in the spatial domain. The contribution in [5] presents a novel algorithm that exploits the statistical anomalies through Laplace modeling of the histograms for detection of anti-forensic contrast enhancement. Experimental results 
demonstrate the effectiveness of the proposed algorithm in detection of contrast enhancements executed both by regular as well as anti-forensics techniques.

The contribution in [6] proposes (i) an efficient algorithm for identifying cloud services based on Quality of Service (QoS) metrics given by the cloud consumer using the decision tree classification algorithm, (ii) an efficient algorithm for cloud service resource registry aiming to enable cloud service providers to register their services with its QoS attributes, and (iii) a cloud service resource discovery for searching in the cloud service registry for cloud services using the Split and Cache (SAC) algorithm. An error regarding the authors names in [6] are corrected in the Erratum [7].

Vehicular Ad hoc NETworks (VANETs) are a highly dynamic network due to the rapid mobility of vehicles. Effective communication in VANETs is enabled by introducing a topology in the network, which can be determined by clustering algorithms. These clustering algorithms must be suitable for highly dynamic networks stabilizing the VANET topology. The contribution [8] proposes an improved variant of the Reputation based Weighted Clustering protocol (RWCP) for VANETs. The RWCP is framed by taking the direction of vehicles, position, velocity, number of nearby vehicles, lane ID, and the reputation of each node into consideration. Simulations and experiments with realistic maps from OpenStreet Maps show the benefits of the proposed method concerning mean cluster lifetime, packet delivery ratio and control packer overhead in comparison with state-ofthe-art approaches.

The authors of the paper [9] propose the extension $k$ repetitive-nearest-neighbor of existing nearest-neighbor heuristics. Starting with a tour of $\mathrm{k}$ nodes the algorithm then performs a nearest-neighbor search from there on. The final result is determined as the shortest tour under all permutations of $\mathrm{k}$ nodes as starting tour of the nearestneighbor search. The proposed approach reaches about $10 \%$ to $40 \%$ above the optimum in experimental results.

Task scheduling aims to balance the workload on a cloud server. Existing approaches are not optimized to handle burstiness workloads containing bursty user demands and provide high-quality cloud services. The contribution in [10] proposes the Threshold Based Multi-Objective Memetic Optimized Round Robin Scheduling (TMMORRS) technique which employs a burst detector to decide on the usage of Weighted Multi-Objective Memetic Optimized Round Robin Scheduling (WMMORRS) for burstiness workload, and the Threshold Multi-Objective Memetic Optimization (TMMO) approach for normal workloads. The experimental results demonstrate that the T-MMORRS technique enhances the scheduling efficiency and also minimizes the energy usage in the cloud in comparison to state-of-the-art approaches.
Recommender systems provide information filtering approaches to suggest relevant items like movies and locations based on dynamic user preferences and interests. The authors of [11] propose a novel Hybrid Location-based Travel Recommender System (HLTRS) through exploiting the ensemble based co-training method with swarm intelligence algorithms to enhance the personalized travel recommendations. Experimental results using real-world large-scale datasets demonstrate a better performance of the proposed HLTRS over existing state-of-the-art recommender systems.

The paper [12] introduces an autonomic prediction model for auto recovery of attacked VM instances in a cloud. The proposed approach to auto recovery and self-healing in the VM's by an intelligent hypervisor offers a continuous progression of any process in the schedule without any job disruption.

Encryption typically secures data in transmission also for cloud computing applications. State-of-the-art encryption techniques encrypt whole data packets in a session using the same session key. Hence once a single packet is decrypted within an attack, all the packets of that session are compromised. The work [13] proposes a packet encryption scheme to ensure that a packet key will only be used once throughout the session, with minimal latency and maximum security for realtime mobile cloud applications.

Various designs of hierarchical interconnection networks (HINs) for massively parallel computer (MPC) systems aim to replace the conventional ones which showed poor performance in scaling the network size. Recently, a Shifted Completely Connected Network (SCCN) is proposed as a new HIN topology. The authors of [14] discuss routing protocols of SCCN and evaluate the time cost-effectiveness factor of SCCN.

We wish all readers of this special issue interesting hours. Please enjoy reading the papers of our special issue.

Acknowledgements The guest editors are thankful to all authors submitting their valuable contributions to our special issue and to our reviewers for their great effort in reviewing the manuscripts, which provided many tips and suggestions to our authors for improving their contributions. We also thank the Editor-in-Chief, Dr. Imrich Chlamtac for his supportive guidance during the entire process.

\section{References}

1. Preethi GA, Gauthamarayathirumal P, Chandrasekar C (2019) Vertical handover analysis using modified MADM method in LTE. Mob Netw Appl. https://doi.org/10.1007/s11036-01901251-5

2. Lavanya S, SaravanaKumar NM, Vijayakumar V, Thilagam S (2019) Secure key management scheme for multicast network using graphical password. Mob Netw Appl. https://doi.org/10. 1007/s11036-019-01252-4

3. Prabha MS, Sarojini B (2019) Online healthcare information adoption assessment using text mining techniques. Mob Netw Appl. https://doi.org/10.1007/s11036-019-01253-3 
4. Rajkumar S, Malathi G (2019) Impact of image artifact and solution to the image quality issues in real time SAR images. Mob Netw Appl. https://doi.org/10.1007/s11036-019-01254-2

5. Bharathiraja S, Rajesh Kanna B (2019) Anti-forensics contrast enhancement detection (AFCED) technique in images based on laplace derivative histogram. Mob Netw Appl. https://doi.org/10. 1007/s11036-019-01255-1

6. Md AQ, Varadarajan V, Mandal K (2019) Efficient algorithm for identification and cache based discovery of cloud services. Mob Netw Appl. https://doi.org/10.1007/s11036-019-01256-0

7. Md AQ, Varadarajan V, Mandal K (2019) Correction to: efficient algorithm for identification and cache based discovery of cloud services. Mob Netw Appl. https://doi.org/10.1007/s11036-01901280-0

8. Joshua CJ, Duraisamy R, Varadarajan V (2019) A reputation based weighted clustering protocol in VANET: a multi-objective firefly approach. Mob Netw Appl. https://doi.org/10.1007/s11036-01901257-z

9. Klug N, Chauhan A, V V, Ragala R (2019) K-RNN: extending NNheuristics for the TSP. Mob Netw Appl. https://doi.org/10.1007/ s11036-019-01258-y

10. J P, Venkataraman N (2019) Threshold based multi-objective memetic optimized round Robin scheduling for resource efficient load balancing in cloud. Mob Netw Appl. https://doi.org/10.1007/ s11036-019-01259-x

11. Ravi L, Subramaniyaswamy V, Vijayakumar V, Chen S, Karmel A, Devarajan M (2019) Hybrid location-based recommender system for mobility and travel planning. Mob Netw Appl. https://doi.org/ 10.1007/s11036-019-01260-4

12. Joseph L, Mukesh R (2019) Securing and self recovery of virtual machines in cloud with an autonomic approach using snapshots. Mob Netw Appl. https://doi.org/10.1007/s11036-019-01261-3

13. Ajay DM, Umamaheswari E (2019) Packet encryption for securing real-time Mobile cloud applications. Mob Netw Appl. https://doi. org/10.1007/s11036-019-01263-1

14. Ali MNM, Rahman MMH, Nor RM, Behera DK, Sembok TMT, Miura Y, Inoguchi Y (2019) SCCN: a time-effective hierarchical interconnection network for network-on-Chip. Mob Netw Appl. https://doi.org/10.1007/s11036-019-01262-2

Publisher's Note Springer Nature remains neutral with regard to jurisdictional claims in published maps and institutional affiliations.

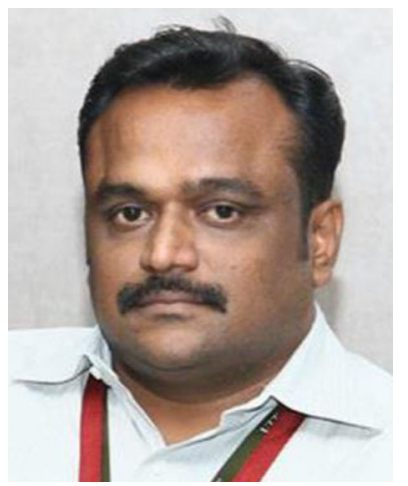

Vijayakumar Varadarajan is currently a Professor in School of Computing Science and Engineering at Vellore Institute of Technology, Chennai, India. He has more than 18 years of experience including industrial and institutional. He also served as a Team Lead in industries like Satyam, Mahindra Satyam, Tech Mahindra and FORD-IT for several years. He has completed Diploma in Computer Technology with First Class Honors. He has completed BE CSE and MBA HRD with

First Class. He has also completed ME CSE with First Rank Award. He has completed his $\mathrm{PhD}$ from Anna University in 2012. He has published many articles in national and international level Journals/Conferences/ Books and Book Chapters with indexing in SCOPUS, SCI and WOS etc.,. He is a reviewer in IEEE Transactions, Inderscience, Elsevier and Springer Journals. He has initiated a number of international research collaborations with universities in Europe, Australia, Africa, Malaysia, Singapore and North America. He had also initiated joint research collaboration between Vellore Institute of Technology and various industries in India and Abroad. He serves as an Editor-in-Chief for EAI Endorsed Transactions on Cloud Systems journal. He is also the Guest Editor for reputed journals of Inderscience, Springer, Elsevier, IOS Press and IGI Global. He also organized several international conferences and special sessions in USA, Vietnam, Africa, Malaysia, UK, Spain and India including IEEE, ACSAT, ISRC, ISBCC, ICBCC etc. His research interests include computational areas covering grid computing, cloud computing, computer networks, IOT, cyber security and big data. He received his university level Best Faculty Award for 2015-2016 from VIT. He is also a member of several national and international professional bodies including EAI, BIS, ISTE, IAENG, CSTA, IEA etc. He is also awarded as EAI Fellow from European Alliance for Innovation Recently.

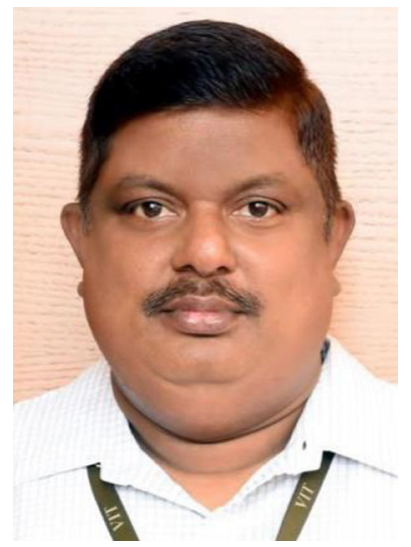

Venkataraman Neelanarayanan received his Master of Science in Computer Science from Madurai Kamaraj University, India in 1995 and $\mathrm{PhD}$ from IT University of Copenhagen, Denmark in 2012. Currently, he is an Associate Professor at VIT University, Chennai, India. Before joining VIT University he has worked as a Scientist at Centre for Advanced Computing (CDAC), India and as a Lecturer in Madurai Kamaraj University, India and its affiliated institutions. His areas of research include distributed computing such as grid and cloud computing, contextaware computing, network management and security, XML-based security technologies and e-communities. He has initiated a number of international research collaborations with universities in Europe, Australia and South Korea as a Research Group Coordinator and Chief Investigator at VIT University. He was instrumental for initiating joint research collaboration between VIT University and industries such as CDAC and DLink. He has published more than 40 papers in various peer-reviewed international conferences and journals. He has organized various national workshops, international conference and symposium. Currently, he is serving as the overall coordinator for research groups at School of Computing Science and Engineering, VIT University, Chennai and six students are pursuing their $\mathrm{PhD}$ under his guidance. He is also the Guest Editor for few journals in Inderscience, Springer and IGI Global. He is editor-in-chief for EAI Transactions on Cloud Systems. He is a member, Editorial Board, International Journal of Scientific Research in Computer Science and Engineering, and International Journal of Applied Science and Engineering, New Delhi Publishers. He received the research award in VIT University for the year 2015 for his achievements, exemplary commitment, dedication and motivation towards research publication during 201516. He is also a member of several national and international professional bodies including ISTE, IAENG, CSTA, SDIWC, IEA, ISTICC, EAI, WASET and IACSIT 


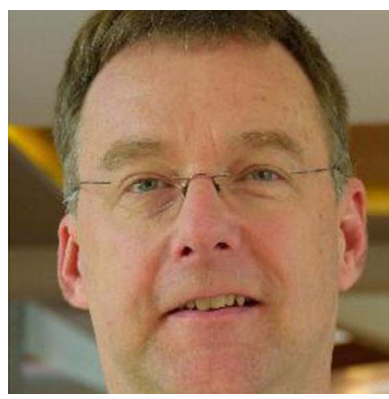

Ron Doyle is division-wide lead architect for enterprise and cloud application design/delivery, focusing on public SaaS, hybrid cloud and on-premise software products. The technical focus is all aspects of cloud and on-premise application/ infrastructure architecture, services, APIs, containerization and DevOps. He is a member of the Board of Directors of the Eclipse Foundation. Before the Broadcom acquisition of CA Technologies, he was Vice President of Continuous Delivery Portfolio Architecture. He drove cross-product integration and product delivery models (SaaS and on-prem) for products in modeling, functional and performance testing, service virtualization and orchestration. Previously, he was a Distinguished Engineer for DevOps transformation across the IBM cloud development organization, focusing on both strategy and hands-on initiatives to address the organization, tools and methodologies needing to enable world-class service delivery. He has been a technical executive, project lead, designer, architect and developer on large scale projects, working with small and large customers at every level of the business. Previously he served as the technical lead for a cross-company organization focusing on key technical and strategic issues across IBM Cloud. His areas of interest include distributed systems and managed resource sharing, focusing on virtualization and cloud computing. He led the IBM involvement in the Open Virtualization Format (OVF) standard in the DMTF. He also spend time working on university accreditation for computer science programs. He has been a program evaluator and team chair for a number of years and currently on the ABET Computing Accreditation Commission Executive Committee and he is a Representative Director for the CSAB Board of Directors.

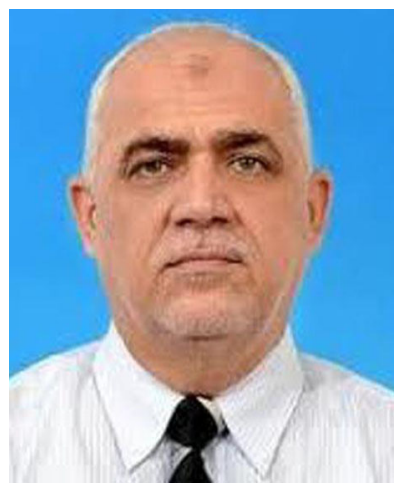

Imad Fakhri Al-Shaikhli is an IEEE senior member, obtained his BSc (Hon) in Mathematics, $\mathrm{MSc}$ in Computer Science from Iraq, and $\mathrm{PhD}$ degree from Pune University, India, 2000. In 2003 he was appointed as the head of department of computer information systems at Alrafidain University College until 2005. Then he joined Gulf universityBahrain January 2006 and appointed as the founding Dean of the college of computer engineering and sciences, during this period he introduced the CCNA certificate to be part of the curriculum which had a strong impact on the students career. In November 2010 he joined IIUM at the Dept. of Computer Science/kulliyyah of Information and Communication Technology. He received the best teacher award in 2011. He is the editor in chief of JACSTR (international Journal on Advanced Computer Science and Technology Research) since 2011 till now and IJPCC international journal since 2015, and the general chair of the international conference on Advanced Computer Science Applications and Technologies) since 2012 till now. He obtained a US patent for his work with his $\mathrm{PhD}$ student on smart traffic light with accident detection system on 2nd Dec 2014. Prof. Imad has published more than 200 articles, conference papers, and book chapters in addition to three books. In addition, he secured more than 10 research grants. He supervised and graduated more 30 PG students. Presently Prof. Dr. Imad is a Professor at the Department of Computer Science and Head of Research at the Kulliyyah of Information and Communications Technology, the International Islamic University Malaysia (IIUM) since 1st November 2013.

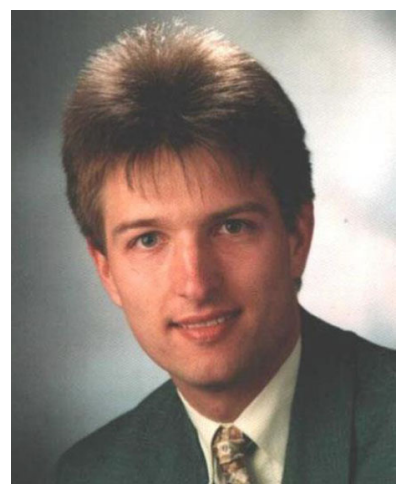

Sven Groppe earned his diploma degree in Informatik (Computer Science) in 2002 and his Doctor degree in 2005 from the University of Paderborn. $\mathrm{He}$ earned his habilitation degree in 2011 from the University of Lübeck. He worked in the European projects B2BECOM, MEMPHIS, ASG and TripCom. He was a member of the DAWG W3C Working Group, which developed SPARQL. He was the project leader of the DFG project LUPOSDATE, an open source Semantic Web database, and one of the project leaders of three research projects, which research on hardware acceleration (via FPGA/GPU) of relational and Semantic Web databases. He is also the chair of the Semantic Big Data workshop series, which is affiliated with the ACM SIGMOD conference (so far 2016 to 2019), and of the Very Large Internet of Things workshop in conjunction with the VLDB conference from 2017 to 2019. His research interests include databases, Semantic Web, query and rule processing and optimization, Cloud Computing, peer-to-peer (P2P) networks, Internet of Things, data visualization and visual query languages. 